\title{
RansomListener: Ransom Call Sound Investigation Using LSTM and CNN Architectures
}

\author{
by \\ Rafeed Rahman \\ 17101502 \\ Mehfuz A Rahman \\ 17101378 \\ Shahriar Hossain \\ 17101370 \\ Sajid Hossain \\ 17101352
}

A thesis submitted to the Department of Computer Science and Engineering in partial fulfillment of the requirements for the degree of B.Sc. in Computer Science
Department of Computer Science and Engineering
Brac University
December 2020

(C) 2020. Brac University

All rights reserved. 


\section{Declaration}

It is hereby declared that

1. The thesis submitted is my/our own original work while completing degree at Brac University.

2. The thesis does not contain material previously published or written by a third party, except where this is appropriately cited through full and accurate referencing.

3. The thesis does not contain material which has been accepted, or submitted, for any other degree or diploma at a university or other institution.

4. We have acknowledged all main sources of help.

\section{Student's Full Name \& Signature:}

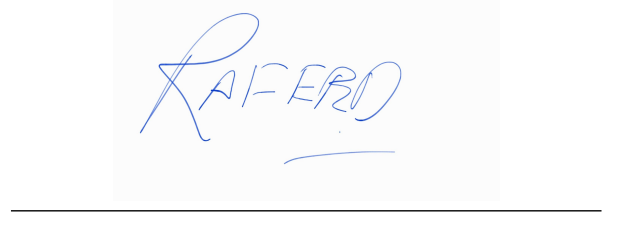

Rafeed Rahman

17101502

$\frac{\text { Shahriar Hossain }}{\text { Shahriar Hossain }}$

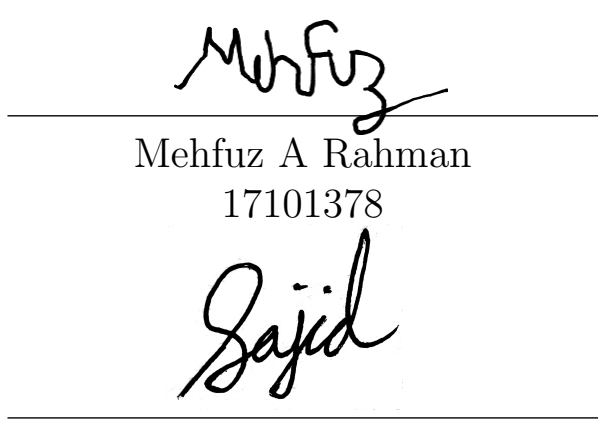

Sajid Hossain 17101352 


\title{
Approval
}

The thesis/project titled "RansomListener: Ransom Call Sound Investigation Using LSTM and CNN Architectures" submitted by

1. Rafeed Rahman (17101502)

2. Mehfuz A Rahman (17101378)

3. Shahriar Hossain (17101370)

4. Sajid Hossain (17101352)

\section{Examining Committee:}

Supervisor:

(Member)

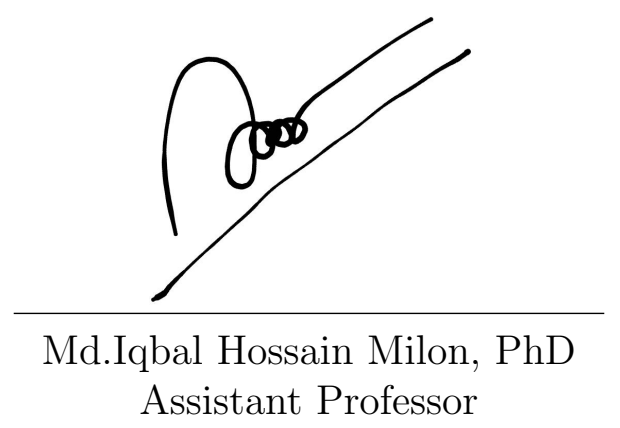

Department of Computer Science and Engineering Brac University

Co-Supervisor:

(Member)

\author{
Mostafijur Rahman Akhond \\ Lecturer \\ Department of Computer Science and Engineering \\ Brac University
}

Head of Department:

(Chair)

Mahbubul Alam Majumdar, PhD

Professor and Chairperson

Department of Computer Science and Engineering

Brac University 
Ethics Statement 


\section{Abstract}

Getting calls for ransoms are common phenomena in kidnapping and abduction related incidents where the life of the victim remains extremely vulnerable. These phone calls are often analyzed in real-time by law enforcement authorities to quickly identify the suspects and get crucial information for quick action. However, it is often difficult to manually analyze those phone calls due to the quality of sounds and the presence of several background noises. Even with much high-end software in their inventory, it is futile to accurately refine the incoming calls as it takes a huge amount of time to declutter the different layers of noises in the call. This paper proposes a model based on deep convolutional neural network and signal processing for automatic classification of crucial sounds in ransom related phone calls. We have proposed LSTM and 2D CNN customized models and compared their outputs with VGG16 and AlexNet. Moreover, this paper also presents a unique dataset of different sounds in terms of voices like male or female and the environmental sounds where the victim might be in which can be a probable clue for investigation purposes consisting of 17650 audio clips collected from verified online sources. Finally, the models produced very high classification accuracy with the accuracy of LSTM reaching around $93.4 \%$.

Keywords: Convolution AlexNET VGG16 LSTM Neural Network 


\section{Dedication}

We have worked hard to ensure that our work possess a great new contribution to the

field Artificial Intelligence. We would like to thank the Almighty for giving us the knowledge, strength, and patience to complete the thesis report. We would also like to thank our parents, teachers, and friends to help us in all possible ways, without which we would not be able to complete the research. 


\section{Acknowledgement}

Firstly, all praise to the Great Allah for whom our thesis have been completed without any major interruption. This thesis has been accepted in the got accepted for publication in the 6th International Conference on Inventive Computation Technologies (ICICT-2021) IEEE. We have updated on paper based on their reviews and sent the final manuscript to them as well

Secondly, to our Supervisor Mr.Md.Iqbal Hossain Milon Sir and Mr. Mostafijur Rahman Akhond Sir for their kind support and advice in our work. He helped us whenever we needed help.

And finally to our parents without their throughout sup-port it may not be possible. With their kind support and prayer we are now on the verge of our graduation. 


\section{Table of Contents}

Declaration $\quad$ i

Approval ii

Ethics Statement iii

Abstract $\quad$ iv

Dedication $\quad$ v

Acknowledgment vi

Table of Contents vii

List of Figures viii

1 Introduction $\quad 1$

1.1 Introduction . . . . . . . . . . . . . . . . . . . 1

1.2 Problem Statement . . . . . . . . . . . . . . . . . . . 2

1.3 Research Methodology ... . . . . . . . . . . . . . 3

1.4 Thesis outline . . . . . . . . . . . . . . . . . 4

2 Related Work 5

3 Methodology 9

3.1 Dataset . . . . . . . . . . . . . . . . . . 9

3.2 Customized 2D Convolutional Neural Network . . . . . . . . . . . . . 12

3.3 Long Short Term Memory . . . . . . . . . . . . . . . . . . . . . . 14

3.4 AlexNET . . . . . . . . . . . . . . . . . . . 16

3.5 VGG $16 \ldots \ldots \ldots \ldots \ldots \ldots$

4 Experimental Setup and Results Analysis 18

4.1 Experimental Setup . . . . . . . . . . . . . . . . . . 18

4.2 Result Analysis . . . . . . . . . . . . . . . . . . . . . 21

5 Conclusion $\quad 26$

$\begin{array}{lll}6 & \text { Bibliography } & 27\end{array}$

$\begin{array}{lr}\text { Bibliography } & 30\end{array}$ 


\section{List of Figures}

1.1 Bar Chart of Age of Victims [3] . . . . . . . . . . . . 1

3.1 Distribution of the data in corresponding classes . . . . . . . . . . . 9

3.2 Mel Frequency Cepstral Coefficient . . . . . . . . . . . . . . . . . . . 11

3.3 Mel Frequency Cepstral Coefficient . . . . . . . . . . . . . . . . . . . 13

3.4 LSTM Model . . . . . . . . . . . . . . . . . . . . . . . . . . 14

3.5 LSTM Model . . . . . . . . . . . . . . . . . . . . . . . . . . . . . . . . . . . 15

3.6 AlexNET . . . . . . . . . . . . . . . . . . 16

3.7 VGG16 . . . . . . . . . . . . . . . . . 17

4.1 Workflow . . . . . . . . . . . . . . . . . . . 18

4.2 Wav Files to MFCC . . . . . . . . . . . . . . . . . . . 19

4.3 The training and validation accuracy and loss of the Customized 2D CNN model . . . . . . . . . . . . . . . . . . . . . . . 21

4.4 Confusion Matrix of the Customized 2D CNN model . . . . . . . . . 22

4.5 The training and validation accuracy and loss of the Customized 2D CNN model . . . . . . . . . . . . . . . . . . . . . . 22

4.6 Training accuracy and Validation Accuracy per Epochs of LSTM MODEL at each 10 epochs of 30 epochs . . . . . . . . . . . 23

4.7 Confusion Matrix of LSTM . . . . . . . . . . . . . . . . 23

4.8 Bar chart representing maximum accuracies . . . . . . . . . . . 24

4.9 Classification Report of VGG16, LSTM, 2D Customized CNN and AlexNet respectively . . . . . . . . . . . . . . . . . . 25 


\section{Chapter 1}

\section{Introduction}

\subsection{Introduction}

Kidnappings and abductions are like exponential functions in the world of crime. A crime that has cemented its position in our world with staggering statistics. According to a paper [1] whose research was conducted on two groups of convicted, around $51 \%$ of males and $57 \%$ of females had records of previous convictions. This record was solely from England and Wales. It has been estimated that kidnapping and asking for ransom money is an industry that is worth USD 1.5 billion [2]. In the global arena there is no availability of systematic acquisition of data but it is estimated that such cases occur between 25000 to 10000 in number annually [3].According to Fig 1.1 mostly young adult and teenagers are the victim of kidnapping.

Age of detected victims, 2007-2017 (\%)

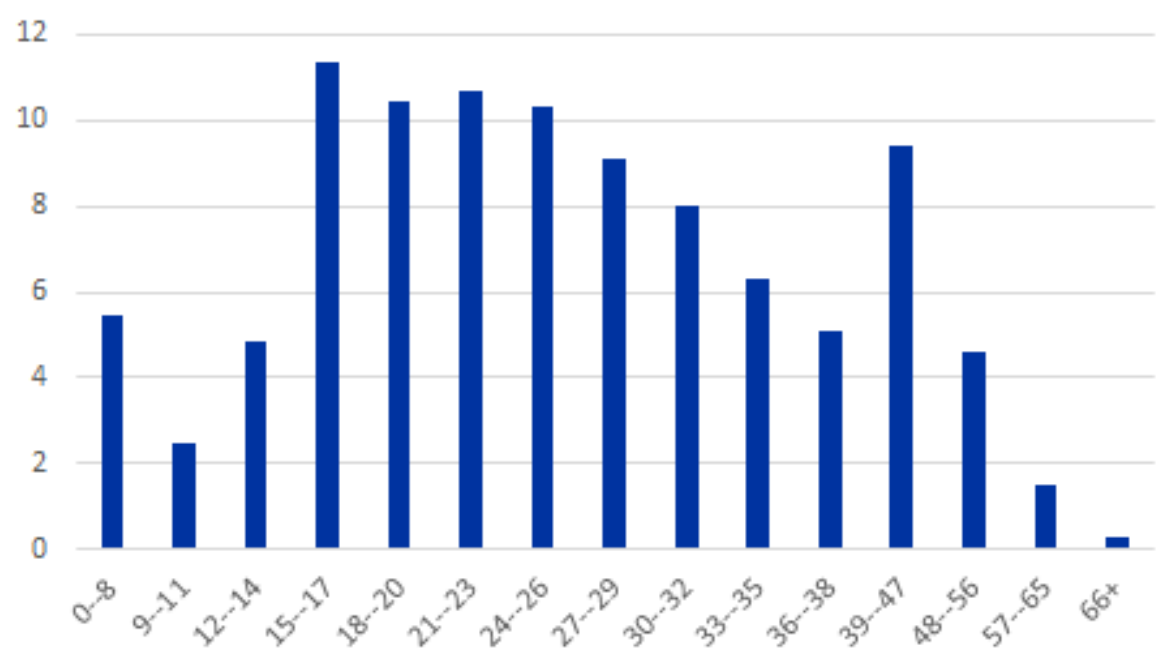

Figure 1.1: Bar Chart of Age of Victims [3] 


\subsection{Problem Statement}

The statistics prove that before these convicts jump to kidnapping and abductions they have already delved into a world of other crimes. It is difficult to track the coordinates of the kidnapper or the abductor from a single call and apprehend them. But always there is more than it meets the eye, in another paper [4] cases sparked major sensation among crowds. Such news painted a common picture into the minds of people regarding kidnappings. But in reality, there is more than just a common vendetta that revolves around such cases.

New technologies have made the criminals deadlier as they use devices that can prevent the law enforcers to tap into the real-time locations of these criminals. Breaking down a single call might not demystify the real intentions of the abductors as often they demand ransom money in their call, but it can surely give away their location which is crucial for the officers in charge and the lives of victims at stake. The background noises in the phone call, if it is identified correctly, can narrow down the search options for the officers, but it is extremely difficult because mostly the criminals call from places that are devoid of any sounds. Some call consists of background noises which are mostly distorted, muffled or shrouded by a lot of other noises. It is almost impossible to separate such a mixture of noises from a single layer of overlapping noises.

On a separate note, [27] deep learning has successfully proved its potential for image processing over the last two decades. One of its significant success was achieved when transfer learning model Alexnet [5] demonstrated its efficacy for image classification tasks on a broader scale. Since then we have seen the entrance of several powerful neural network architecture like VGG16 [6] and outstanding success for recognition of images in practice like medical diagnosis $[7,8]$, facial detection $[9$, $10]$, object detection with unmanned area vehicle imagery $[11,12]$ and many more. Although much of the attention and noteworthy success for deep learning was in the domain of image processing, one cannot simply disregard the unique potential of deep learning for audio processing since many modern intelligent systems like virtual assistants Alexa introduced by Amazon, Siri by Apple, and Google Home are products of audio signal processing with deep learning [13].

Furthermore, the importance of audio processing using deep learning was demonstrated by many researchers. For instance, environmental sound classification using deep convolution neural networks [14], classification of heart sound [15], and even sounds of birds [16] which is often difficult to classify.

In many crime incidents related to abductions, we have seen that the lack of time to track the kidnappers and the inability of the law enforcement officers to track the kidnappers in due time breeds catastrophic results. Often the presence of several sounds backed up by distortion caused by the voice devices produces multi-layer sounds that hide or cloaks the prime sounds that can disclose the location of origin of the call. We did this research because we wanted to work on something which can actually solve the problem of our society which is recurrently happening. Not just locally but abduction cases are very common even in the global scenario. 


\subsection{Research Methodology}

Considering the problem statement and technology stated above, in this paper, we have proposed a unique strategy to apply deep learning for the classification of sound in ransom based phone call recordings to help the law enforcement authority in abduction related crime investigations. To be precise, we have proposed and demonstrated a deep convolution neural network architecture which is capable of classifying ransom based phone calls in abduction related crime incident. Furthermore, we have used our dataset for training and testing which consists of around 8000 sounds commonly present in ransom-based phone call.

We have proposed LSTM and 2D CNN customized models and compared their outputswith VGG16 and AlexNet. Moreover, this paper also presents a unique dataset ofdifferent sounds in terms of voices like male or female and the environmental soundswhere the victim might be in which can be a probable clue for investigation pur-poses consisting of 17650 audio clips collected from verified online sources. 


\subsection{Thesis outline}

The subsequent of the paper is organized as follows: Section II contains the literature of the latest research papers of the topic and the background information of the algorithm that we have used. Section III contains the details about the preparation of the dataset and the architecture of the proposed models. Finally, the results section contains the visualizations of the observations in terms of accuracies and losses of each model. 


\section{Chapter 2}

\section{Related Work}

For the past few years, a variety of works has been done on sound signal classification using machine learning and its subset deep learning. For example, [17] proposed a unique method for the identification of underwater vehicle noises, engendered by faulty mechanical setup, using SVM and incremental learning. This paper applied Example- Incremental Learning and Class-Incremental Learning with SVM as a classifier. Their proposed classifier is dynamic, adjustable, and can learn new parameters of information and store them as well.

The authors of this paper [28] tried to come up with a system that would detect pneumonia and could be used in remote areas. They used an x-ray image dataset previously used by Wang et al. (2017, as cited in Varshni et al., 2019) that comprises of 112,120 chest X-ray but represent fourteen different thoracic diseases. The authors used Xception, VGG16, VGG19, ResNet-50, DenseNet-169, and DenseNet121 for feature extraction. For classification purposes, they used Support Vector Machine (SVM), Random Forest, K-nearest neighbours, and Naive Bayes. They observed the best results with an Area Under the Curve (AUC) of .8002 while using DenseNet-169 and SVM.

Research [18] suggested a model for the classification of in-vehicle noise via the Deep Beamforming Network model. To be precise, this paper introduced a beamforming method to get intra-inter channel spatial features by the optimal beamforming weights which include Direction-of-Arrival (DOA) to manipulate the reflective and refractive nature of in-vehicle noise. [19], on the other hand, proposed a method for classification of environmental sound based on feature extraction and time-frequency. They made use of Multi SVM for the classification of environmental sound effects in the final step of their model. [20] Also worked with the classification of environmental sound however they applied deep convolution neural networks for their purpose. Their work also provided an important suggestion by describing the need for data augmentation for avoiding a lack of data problems and to analyze the impact of augmentations on the output of CNN.

Other noteworthy signal processing works using deep learning include [21] that introduced a model based on a pre-trained deep convolution neural network model for classification of the exact location of snoring sound in the human body. Just like most papers in this domain they also worked on spectrogram Creation and Deep 
Spectrum Feature Extraction as the initial steps of their model before training their designed system. On a separate note, there is some significant work that showed different ways of audio forensic techniques are also very relevant for this research.

In this paper[29], the authors have determined the age and gender of a speaker supported a hybrid architecture of Weighted Supervised Non-negative Matrix Factorization (WSNMF) and General Regression Neural Network (GRNN) for law enforcing agency to work out the various characteristics of a speaker from recorded voice patterns (telephone conversations or tape recordings), which facilitate the identification of a criminal for investigation of crime cases like kidnapping, threatening call or false alarms. In this paper, the speaker's gender is decided however, the age is estimated which is difficult to work out as this relies on multiple factors. Firstly because the perceptual age and age varies greatly, secondly, the voice pattern varies greatly on certain parameters like weight, height and emotional condition and lastly due to developing a strong age recognition system requires a labeled, wide age-range and balanced database.

Previous work includes a way to incorporate Support Vector Machine (SVM) with a Gaussian Mixture Model (GMM) supervector to classify speakers into seven agegender categories with the utilization of Mel Frequency Cepstral Coefficients (MFFCs) as features. However, this may require a high number of dimensions in GMM which was solved by employing a SVM classifier with Gaussian mixture weight supervectors which have a lower dimensions. This process worked rather well in determining the gender but couldn't give accurate age estimation of the speaker. The second alternative was to supply the utilization of unsupervised Non-negative Matrix Factorization (NMF) rather than using Gaussian mixture weight supervector which increased the accuracy aged estimation but on the opposite hand decreased the accuracy of gender determination. This paper developed the utilization of WSNMF and GRNN, an hybrid architecture to extract and concatenate Gaussian mixture weights to make a supervector for every speaker within the database which was used as a training data set.

WSNMF is Weighted Supervised Non-negative Matrix Factorization which is a mixture of WNMF and SNMF. NMF is Non-negative Matrix Factorization which is a famous machine learning algorithm that is used in word word recognition, sound source separation and spam filtering. Soon different versions of the NFM i.e. WNMF and SNMF are made to solve real-life problems. On the other hand, GRNN is General Regression Neural Network which is a universal function approximator. This GRNN has two benefits to use than a normal NN because firstly it does not require iterative learning algorithms but instead, it has a one pass and fast learning. Secondly, it does not require a lot of data for training unlike those needed in NN's back propagation. It can be used when the data is limited.

For the training phase, the info set is split into two parts namely training and testing data set. and therefore the training data set is further divided into primary and secondary patterns which are wont to train WSNMF and GRNN respectively. For the testing phase, the testing data is fed into the proposed model and therefore the refore the age-gender category of any unseen speakers is recognised by the trained 
WSNMF (by using the Gaussian mixture weight supervectors of the first training set) and the age is estimated by the trained GRNN (on the secondary training set along side trained WSNMF). The speakers of the info set were classified into six age-gender categories namely Young Male (YM), Young Female (YF), Middle Male (MM), Middle Female (MF), Senior Male (SM), Senior Female (SF). The gender detection of the proposed WSNMF was $96 \%$ accurate and performs better than guessing. On the opposite hand, the accuracy aged estimation or Mean Absolute Error (MAE) using the hybrid GRNN method was 7.48 years which was $15 \%$ better than the MAE of the typical true age of all speakers which was 8.88 years.

In this paper, a way of detecting snore sounds using image classification convolutional neural network (CNN) by extracting features of the audio spectrogram is proposed by the authors. Here they need used two different common CNNs called AlexNet and VGG19, which are used as feature vectors in fully connected networks [30].Snoring is an Obstructive apnea (OSA) which has the second highest prevalence of all sleep disorders. This OSA can cause attack or other heart diseases, however, this will be treated if the precise location where snoring occurs, , i.e. site of obstruction and vibration, is detected. This vibration are often from four different sources namely velum $(\mathrm{V})$, oropharyngeal lateral walls $(\mathrm{O})$, tongue $(\mathrm{T})$, and epiglottis $(\mathrm{E})$, briefly V.O.T.E. The system proposed during this paper is to extract deep spectrum features from spectrogram plots using pre-trained CNN. Then a classification is formed on the extracted feature vectors that are trained using Support Vector Machine (SVM) classifiers. In other words, the subsequent are the steps of the proposed system

Spectrogram Creation Deep Spectrum Feature Extraction Classification

Python package matplotlib is used to create plots of the sound spectrum and a colour mapping is created to represent the snore samples. Three different 'standard' spectrogram colour mappings were used for low, mid and high ranges: jet varies from blue to green to red; gray varies from black to grey to white; and finally, viridis varies from blue to green to yellow, respectively. These spectrograms are then used as input into the CNN after being scaled and cropped for AlexNet and VGG19. During the deep spectrum feature extraction, the CNN is used to extract features of the spectrums. AlexNet which is the first large CNN to have been applied and has shown great success. VGG19 is also popular for creating CNN descriptors of images. Finally the spectrogram is classified into four classes Velum (V), Oropharyngeal $(\mathrm{O})$, Tongue $(\mathrm{T})$, and Epiglottis (E) using linear SVM as it performs quite well with small amounts of training data.

After conducting the experiment, the unweighted average recall (UAR) of the development phase was $44.8 \%$ and that of the testing phase was $67.0 \%$ which both performed better than $40.6 \%$ and 58.55 respectively. Thus, in comparison to VGG19, AlexNet performed better in extracting the features.

For instance, in paper [22] the authors have determined the age and gender of a speaker employing a Weighted Supervised Non-negative Matrix Factorization (WS- 
NMF) and General Regression Neural Network (GRNN) for law enforcing agency where characteristics of speaker has been determined from voice patterns (telephone conversations or tape recordings), which facilitate the identification of a criminal for crime investigation. [23], on the opposite hand proposed an automatic system that authenticates and differentiates between the fake and real audio as a controlling tool for detective and preventive purposed in real-world circumstances, like fake evidence, protecting copyright, and data hacking. Their proposed system was reported as $10 \%$ accurate on two different environments channels and $99 \%$ on the classification of three different environments. 


\section{Chapter 3}

\section{Methodology}

\subsection{Dataset}

We have gone through several documentaries of kidnapping to gather ideas about the types of sounds from ransom calls that generate distinct clues about the areas from which the ransom call originates. Background sounds can be a huge clue for the investigation department and may have a conclusive result. After scrutinizing through those documentaries we have created the following classes of our dataset - Adult Voice, Breathing, Crying Babies, Crying Man, Crying Woman, Factory Noises, Forest Sounds, and Sounds from Inside a Car, Ship Sounds, Market Area Noises, Police Siren, Screams, Ship Sounds Teenager Voice, and Adult Voice and inside a train represented in Fig 3.1 All these sounds have been definite clues of the kidnapping.

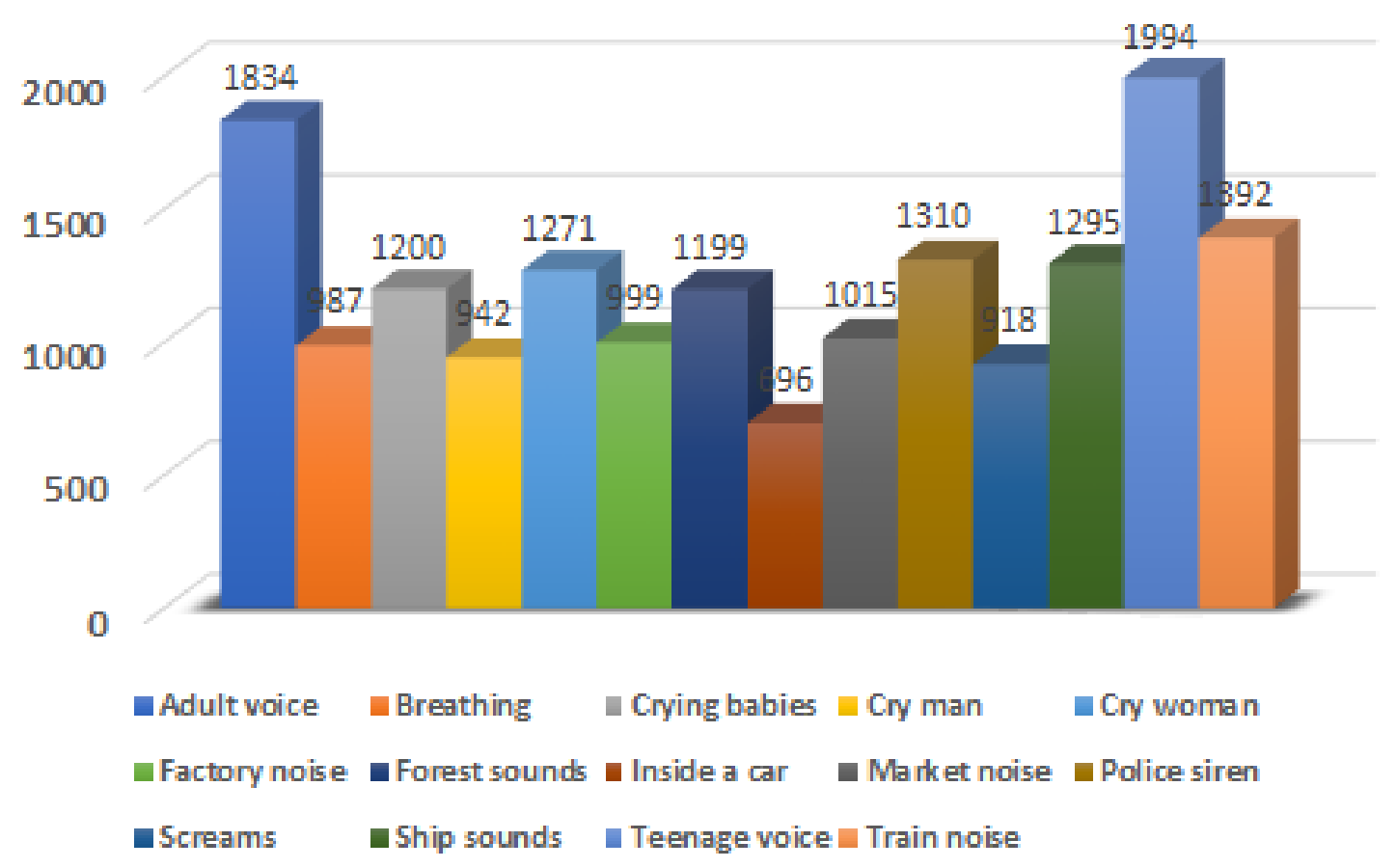

Figure 3.1: Distribution of the data in corresponding classes 
All these sounds are gathered from authentic sources such as Google Audio Datasets, verified YouTube Channels such as Discovery Channel, and other sources. Adult voice - A presence of an adult voice means there are multiple numbers of people who are involved in the abduction.

Breathing sound can alert the detectives of the condition of the captors or of the abducted person. If the breathing is heavier then it can be assumed that they are in a confined place where oxygen concentration is low. Crying babies - The kidnapped individual is a baby, if a collection of crying babies is detected then it indicates that the phone location is near a hospital or parks or any site filled with babies. Or, in the worst-case scenario, the location is somewhere where the kidnappers have held hostage several abducted babies. Crying men - Suggests possibilities that there are abducted men as well from the place where the kidnapper call originated and the men have been tortured. This indicates that the people behind the crime are no petty goons. Crying women - Invites to assume theories that there might be women who are captured to be sold to foreign countries or held to be used for other ransom demands. This suggests that the people behind the crime are running big rackets and must be hidden in an ultra-secure lair.

Factory noises - This means the location is anywhere near a factory or maybe in a factory itself. The genre of the sound will confirm the type of factory it is, further narrowing down the search. Forest sounds - Noises from insects and animals along with the rustling of leaves can confirm the source of communication to be from a forest. Car interior sounds - The clanging sounds of car doors opening and closing or any noise coming out of the motors that move the side glasses can confirm the call source to be from a car.

Market noises - Background sound consisting of commotion and high amplitude of public noises will ensure that the spot is anywhere near a market place. Police siren - Siren like this would allow the detectives to realize that the captive is being held in place which either has a police station nearby or police patrols. Screams Screams would indicate a sign of more captives being held or that the captives are in the same location as the abductors and are trying to break free, not trapped in a separate place. Sounds from the ship - Ports and ship terminals always produce a distinguishable sound, any sound similar to this would make the search narrower by making it lucid that the location of the callers is originating from ports and terminals. Train noise - Loud amplitude noise generated by people and occasional train trumpets and air whistles can reveal that the phone call is originating from a train station. 


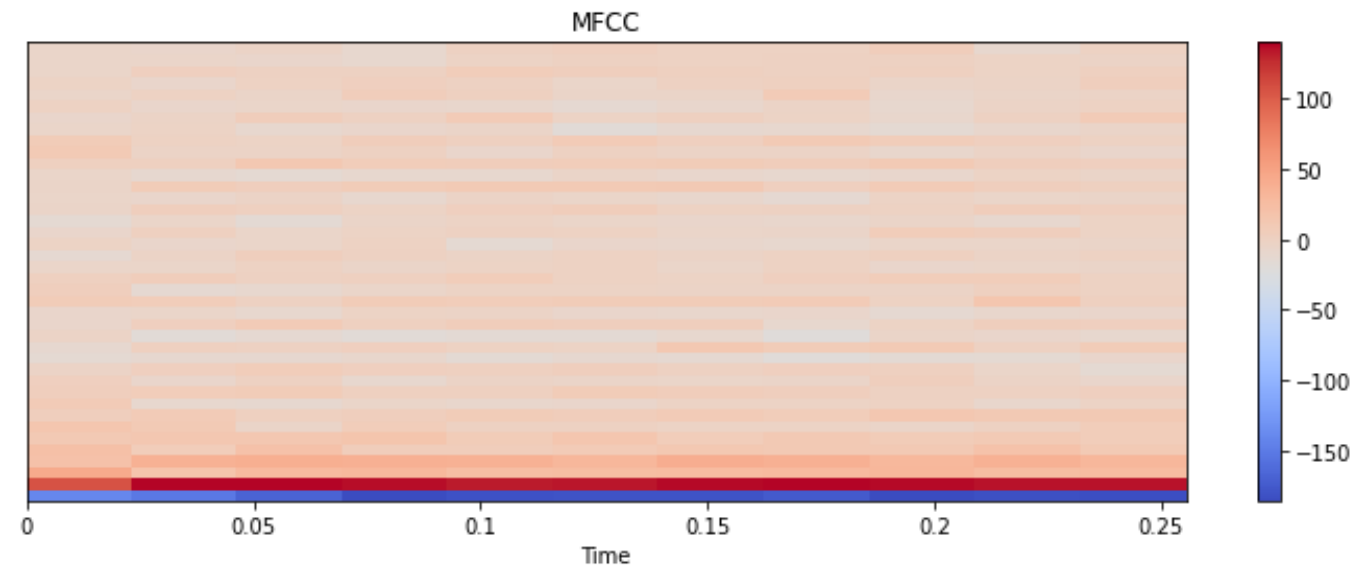

Figure 3.2: Mel Frequency Cepstral Coefficient

After gathering all the sounds, with the help of the AudioSegment package from Pydub, a Python function was defined which will access all the sound files sequentially and clip the sounds into 3 seconds audio clips. Thus finally we have created a dataset containing 17650 sound clips in total for all the classes.

Then using those audio clips we have generated features in terms of Mel Frequency Cepstral Coefficients. In the analysis of sound signals, the Mel Frequency Cepstral Coefficient (MFCC) vectors are the most accepted tools. The MFCC is a portrayal of a short-term logarithmic power spectrum that has been under a linear Cosine transformation on a non-linear Mel scale of Frequency [24]. The derivations are done generally using Fourier analysis of a signal and then using triangular overlapping windows, mapping of the powers of the spectrum onto the Mel scale, where logarithmic values of each power are taken using discreet cosine transformation of those powers. The MFCCs are the resultant amplitudes of the signals. Fig 3.2 shows the mfcc representation. 


\subsection{Customized 2D Convolutional Neural Network}

The convolutional Neural Network that we have proposed for this Ransom Call investigation purpose is a sequential model containing 120 filters in each of the 4 convolution layers respectively. The first convolution layer contains filters of (4X4) dimensions and the rest of the layers contain (3X3) dimensions. Fig 3.3 represents a visualization of our CNN model.

The batch Normalization technique after each layer ensures that the learning rate can be kept high. It ensures the model can achieve faster accuracy with lower epochs [25]. Another regularization technique that we have used is to drop out in the dense layer. The dropout technique ensures that some of the neurons are disconnected randomly that is the influence of those disengaged neurons in activating the next neuron and also weight updating in the back propagation is stopped. This ensures that overfitting or under fitting problems are avoided. The dropout rate is 0.5 .

Dropout enhances the capability of neural networks in all sorts of supervised learning works such as visual classification, audio classification, etc. [26]. The activation function in each of the layers that we have used is ReLU activation functions. The ReLU functions is as follow

$$
\text { Output }=\operatorname{maximum}(0, y)
$$

It chooses any value other than negative values. Thus it clips any negative values and hence enhances the training speed of the neural network. The activation function in the output layer is the Softmax function.

Categorical Cross Entropy is the loss function that we have used The comparison between the actual distribution and the predictions are done by Categorical Crossentropy where the real class has probability one and the rest 0.

The output from the convolutional layers is then flattened and then passed as input to the dense neural layers of 2056, 1025 neurons, and the final 14 neurons in the output layer. 


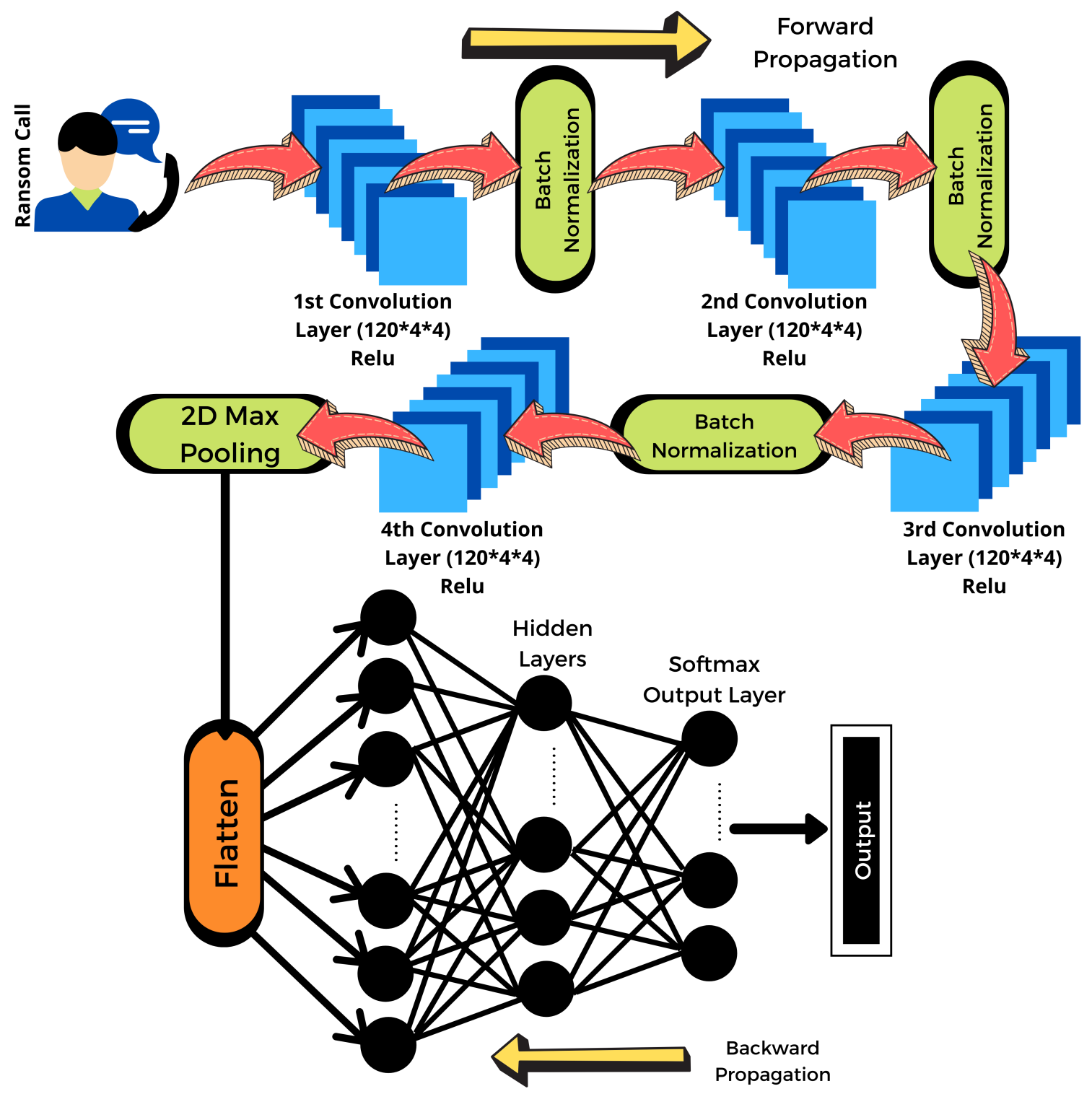

Figure 3.3: Mel Frequency Cepstral Coefficient 


\subsection{Long Short Term Memory}

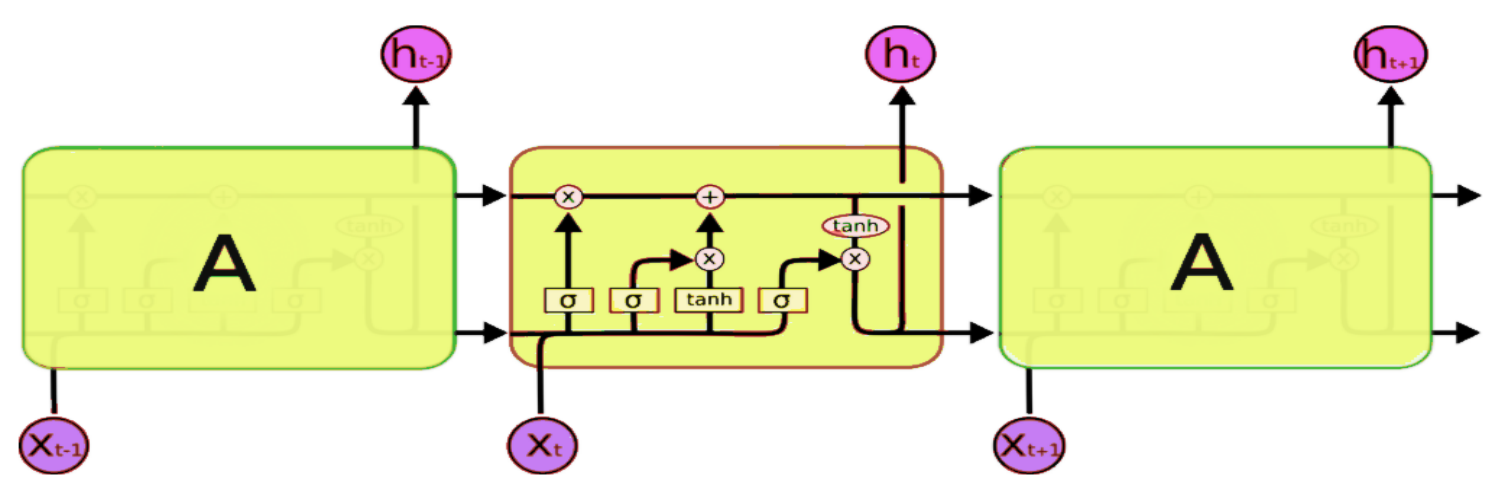

Figure 3.4: LSTM Model

The main key of the LSTM is the cell state which the straight channel at the top of the diagram which can easily allow the information to pass between the input and output state as it is or add or remove any information which is controlled by the gates. The lstm basically has 3 gates [23].

The first step of lstm is to figure out which information is to be removed from the cell state. The $\mathrm{x}(\mathrm{t})$ and the $\mathrm{h}(\mathrm{t}-1)$ are then passed through the sigmoid function. The sigmoid output determines whether to keep any information or remove it. The next step has two parts. The initial sigmoid layer known as the input gate layer to determine the value that will be updated and the other Tanh layer creates an array of new values that might be included in the cell state. These two are combined to generate the updated state.

Finally, the decision about what will be the output is taken. This output will be based updated cell state. First, the sigmoid layer parts of the cell state getting to output. Then, the cell state through Tanh (to push the values to be between 1 and1) and multiply it by the output of the sigmoid gate, to ensure only the decided a part of the cell is that the desired output.

Unlike the previous customized 2D CNN model where we have used 3-dimensional input as the input of the CNN model, here the mfcc vectors are flattened and reshaped to 2 dimensions for the input of the LSTM layer. Fig 5 represents the view of our LSTM model. The input shape is $(440,1)$ for the first LSTM layer with units $=220$. The dropout is assigned a value of 0.05 and the recurrent dropout is 0.20. The return Sequence for the first LSTM layer is set as TRUE. For the second LSTM layer, the value of the unit is 150 and the dropout and the recurrent dropout value are kept the same. The return sequence is again kept as true as the previous LSTM layer and the third LSTM layer has units of 64 with the same dropout value. However, the return sequence is kept as False.

The output from the third LSTM layer is then given as input to a dense Neural Network with 128, 64, and 28 neurons of each layer and the final output layer of 


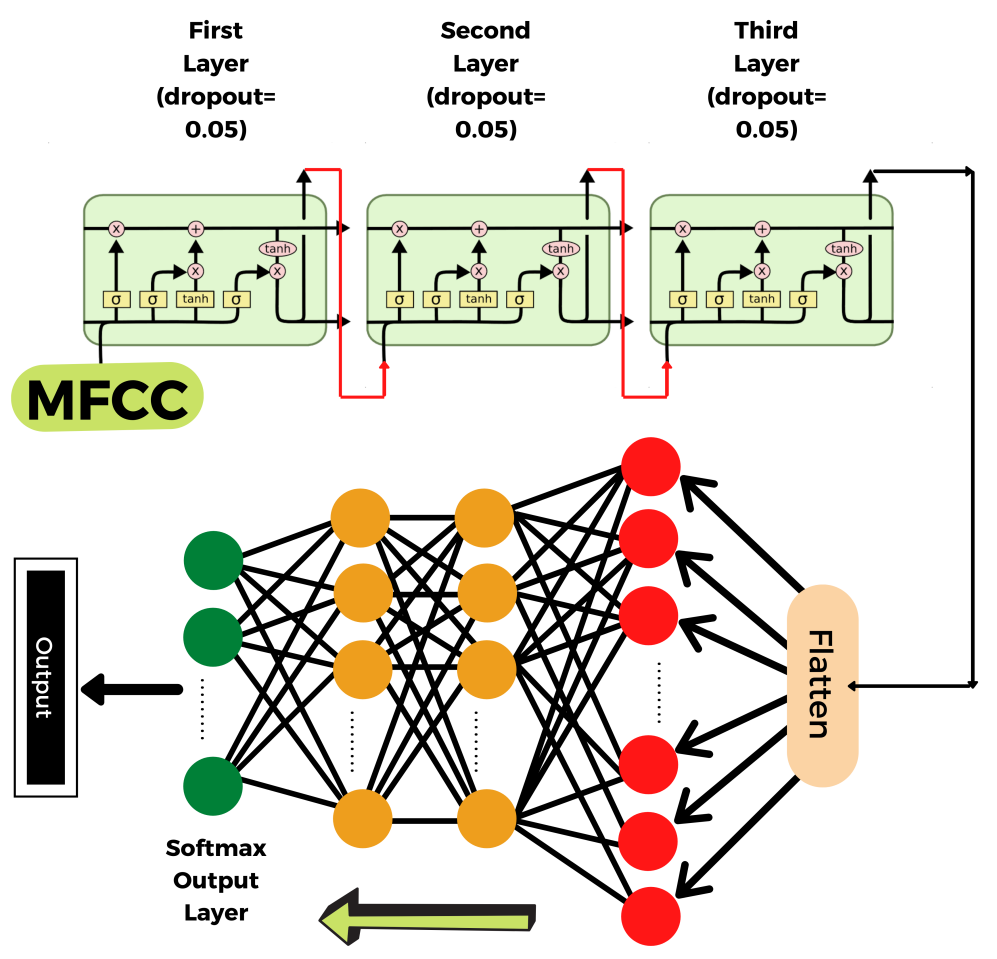

Figure 3.5: LSTM Model

14 Neurons. The activation function in each layer is the ReLU activation function like the previous CNN model. The optimizer that we have used is Adam optimizer which is an optimization algorithm that has an adaptive learning rate. It has an advantage that the algorithm signifies the ability of the adaptive learning rate method to determine the learning rates of individual parameters. 


\subsection{AlexNET}

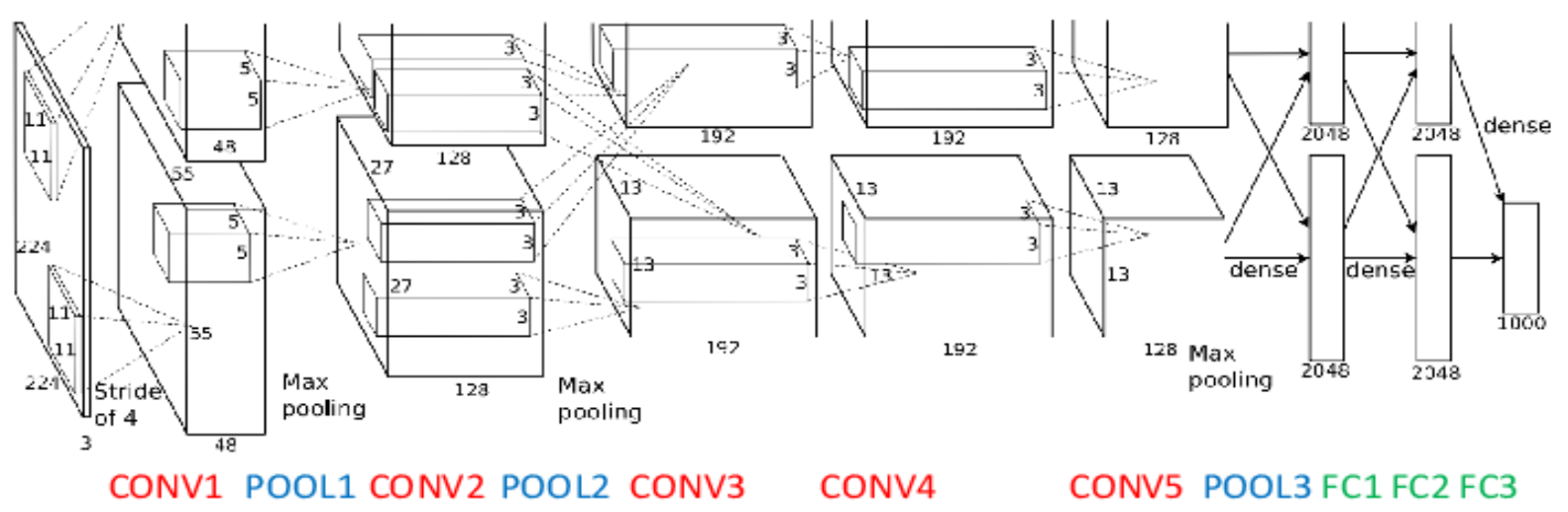

Figure 3.6: AlexNET

The structure of the AlexNet architecture is such that that it consist of eight layers where the first five layers are convolution layers and the remaining three are dense layers that are fully connected. In our model, we have followed the same architecture that was proposed by Alex Krizhevsky with the first Convolution layer has size of [11 X 11] with 96 kernels and 4 strides for filtering the input image of [224 X 224 $\mathrm{X}$ 3]. After Max Pooling $2 \mathrm{D}$ of pool size $=\left[\begin{array}{lll}2 & \mathrm{X} & 2\end{array}\right]$, it is again fed into a second Convolution that uses 256 kernels with a size of [5 X 5] for filtering as shown in Fig 3.6. There are again layers of Convolution where 3rd and 4th layer convolution has a size of [3 X 3] with 384 kernels and the 5th layer also has a size of [3 X 3] with 256 kernels. Then after Batch Normalization, the vector is forwarded to dense layers with 4096 units, 4096 units, and 1000 units and finally an output layer containing 14 units with softmax activation. After the training and testing of the model with our dataset, we have visualized the training accuracy and testing accuracy in the results section. 


\subsection{VGG 16}

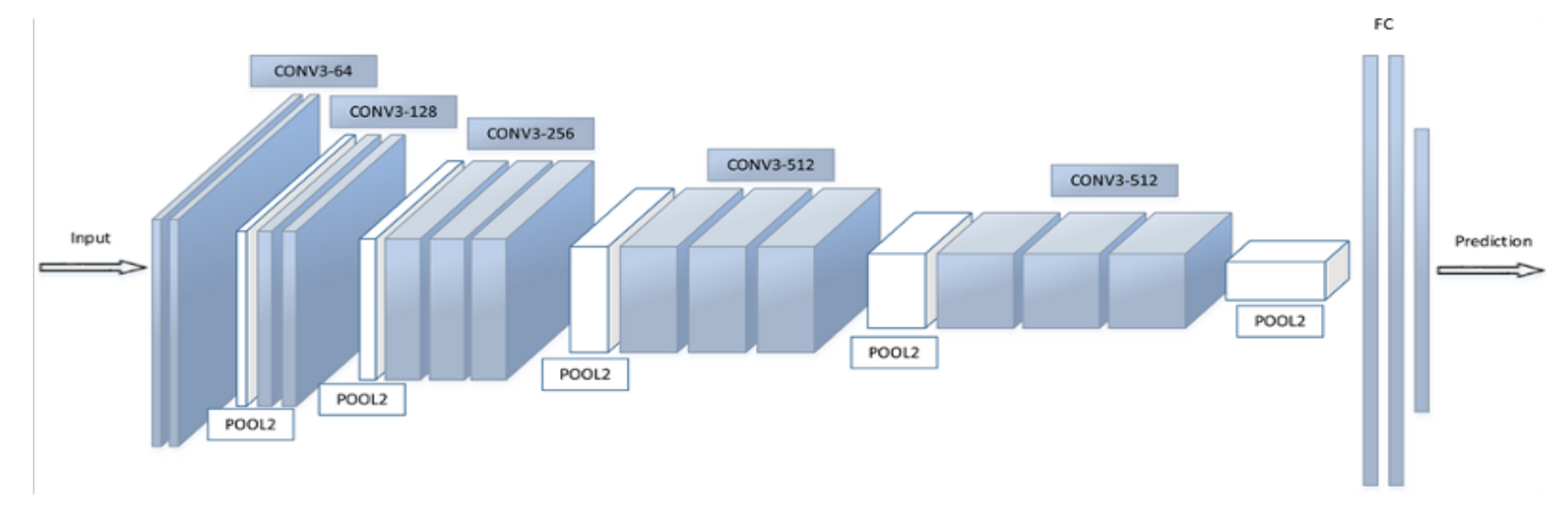

Figure 3.7: VGG16

VGG 16 CNN model had a huge success in ILSVR. Vgg16 is taken under consideration to be one of the only vision model architectures of all time. one of the foremost distinctive features of VGG16 is that instead of having an outsized number of hyperparameter it's targeting having convolution layers of 3x3 filter with a stride of 1 and always having the same padding and maxpool layer of $2 \times 2$ filter of stride 2. Vgg16 follows this distinct exhibition of convolution and max pooling layers consistently throughout the entire architecture. within the top, its $2 \mathrm{FC}$ (fully connected layers) followed by a softmax for output. The 16 in VGG16 means the architecture has 16 layers that contain weights. This network could also be an enormous network and its about 138 million parameters [22].

Although both these transfer learning models have been used for image classification with 224X224 dimensions input but we are using it for the sound detection purpose. With the outputs of these two transfer learning models we are going to compare our RanNET and LSTM Network Model in the results section. 


\section{Chapter 4}

\section{Experimental Setup and Results Analysis}

\subsection{Experimental Setup}

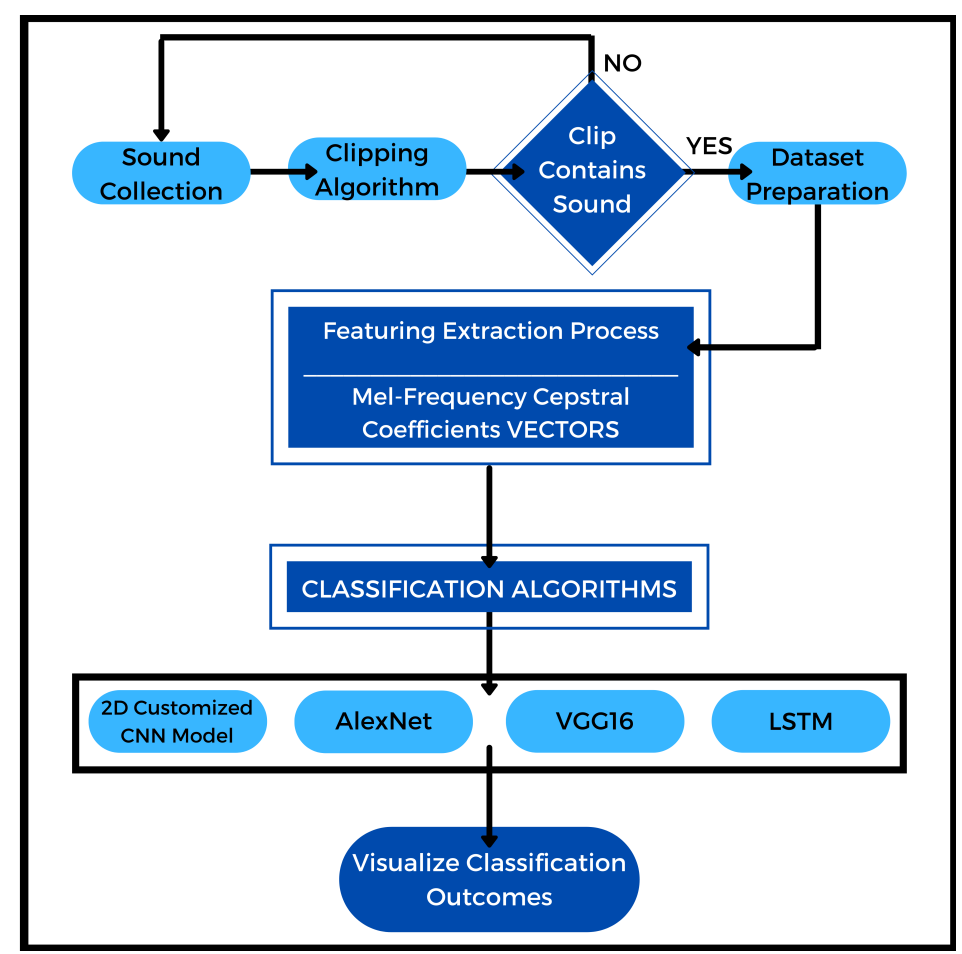

Figure 4.1: Workflow

We have used Google Colab for running all the models. Colaboratory is a Google research project created to help for Artificial Intelligence research purposes. It a notebook based on a Jupyter environment removes any complex setting up processes and fully run in cloud-based.

The research commenced with gathering sounds for the classes that we have prepared which include Adult Voice, Breathing, Crying Babies, Crying Man, Crying Woman, Factory Noises, Forest Sounds, and Sounds from Inside a Car, Ship Sounds, Market Area Noises, Police Siren, Screams, Ship Sounds Teenager Voice, and Adult 
Voice and inside a train. Then after gathering the sounds, the clipping function is used to convert the sounds into 3 seconds audio clips.

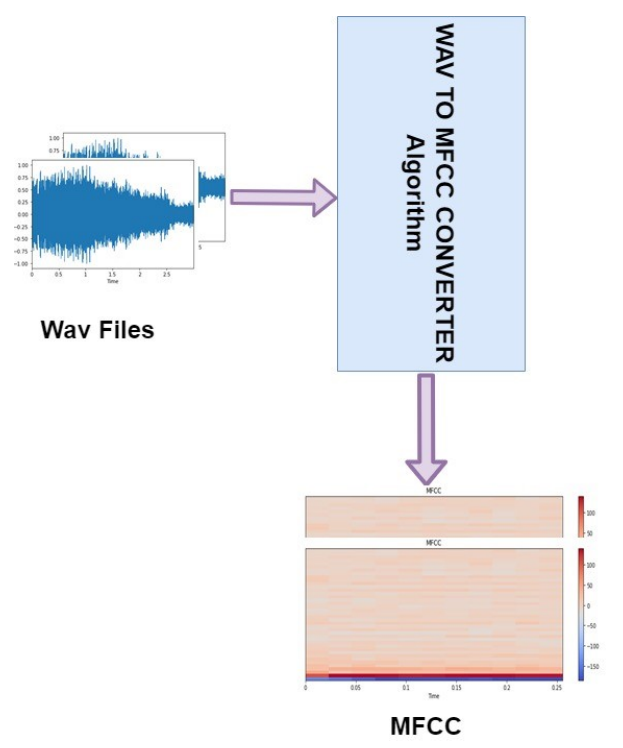

Figure 4.2: Wav Files to MFCC

After preparing a dataset of 17650 audio clips, MFCC Vectors [MFCC] with Librosa Package as shown in Fig 4.2 are generated.This feature is one of the foremost important methods to extract a feature of an audio signal and is used majorly whenever working on audio signals. The mel frequency cepstral coefficients (MFCCs) of a symbol are alittle set of features (usually about 10-20) that concisely describe the overall shape of a spectral envelope. we've written the wavtoMFCC function to convert to urge the mfcc vectors.

Initially, the audio file is loaded with librosa.load function where the parameters passed are the location of the clip, sampling rate with the value assigned 8000 and duration of 1.024 seconds, and returns the audio time series and the sampling rate. Then the librosa.util.normalize was used to normalize the audio time series generated. Hence, the mfcc vectors are generated using a function with parameters input is a normalized audio wave, sampling rate, number of mfcc to return are 40, hop length $=0.048 \mathrm{X}$ sampling rate which is cast to an integer and the output from the function is mfcc.

librosa.display is employed to display the audio files in several formats like wave plot, spectrogram, or colormap. Waveplots allow us to know the loudness of the audio at a given time. The spectrogram shows different frequencies playing at a specific time along side its amplitude. Amplitude and frequency are important parameters of the sound and are unique for every audio. 
The MFCC vectors are then fractioned into training and validation data in 7:3 respectively into X-Train, X-Test, Y-Train, and Y-Test which is then used to train the customized 2D CNN model. The outcome of the customized 2D CNN in terms of Training and validation accuracy and losses are visualized in the results section.

For training the recurrent LSTM model, we have flattened the Mel-Frequency Cepstral Coefficient vectors and then reshaped it into an array of dimension (34904, $40 * 11$, and 1) for training the LSTM model. Then this updated dimension array is used to train the LSTM model. 


\subsection{Result Analysis}

The proposed models are trained with the vectors and the outcome of training and the accuracy of prediction is compared with VGG16 and AlexNet model.
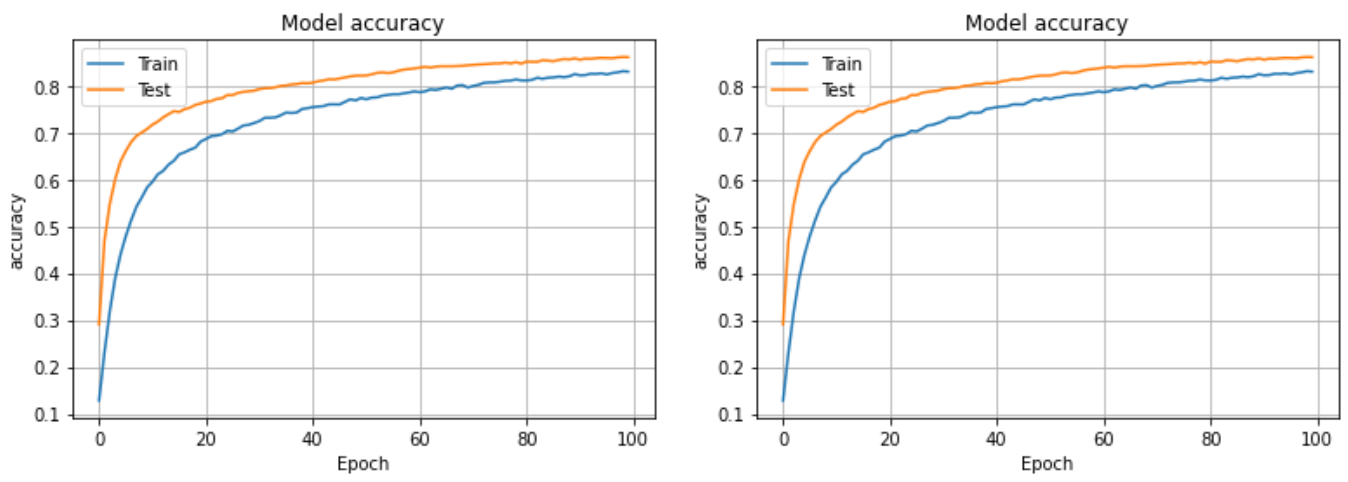

Figure 4.3: The training and validation accuracy and loss of the Customized 2D CNN model

The customized 2D CNN model is trained for 100 epochs possessing a batch size of 32. The training set is split into training and validation with validation is $10 \%$ of the size of training data. The variation in training and testing is visualized using the curves representing Training and validation accuracy VS Epoch and the Training and validation Loss VS Epoch in Fig 4.2. As we can see the accuracy is increasing exponentially and the loss is decreasing exponentially.

The confusion matrix in Fig 4.3 reflects the accuracy of the detection of classes. Since the figure has a dense color diagonal line, it shows that the customized CNN model is successful in detecting the maximum number of the testing data to their particular classes that they belong to. Fig 4.4 portraits variation of training and validation accuracy of the VGG16 model AlexNET model per epochs. The training accuracy has gradually increased to $95 \%$ exponentially and the validation accuracy reaching around $90 \%$ of the VGG16.

Next, the curves in Fig 4.5 indicate the training and validation accuracy variations over every 10 epochs of the LSTM. This variation is more linear than exponential and the maximum training accuracies reaching $93.4 \%$.

The confusion matrix in Fig 4.6 again proves the accuracy of the detection of classes is higher in the case of True positives for the LSTM model when we tested it with our test data.

To observe this maximum accuracies in terms of training and validation, we have used a bar chart as visualization in Fig 4.7. The bar chart portraits that all the models have performed well in terms of training and validation and the two particular models which have displayed remarkable performances are - VGG 16 and the proposed LSTM model. 


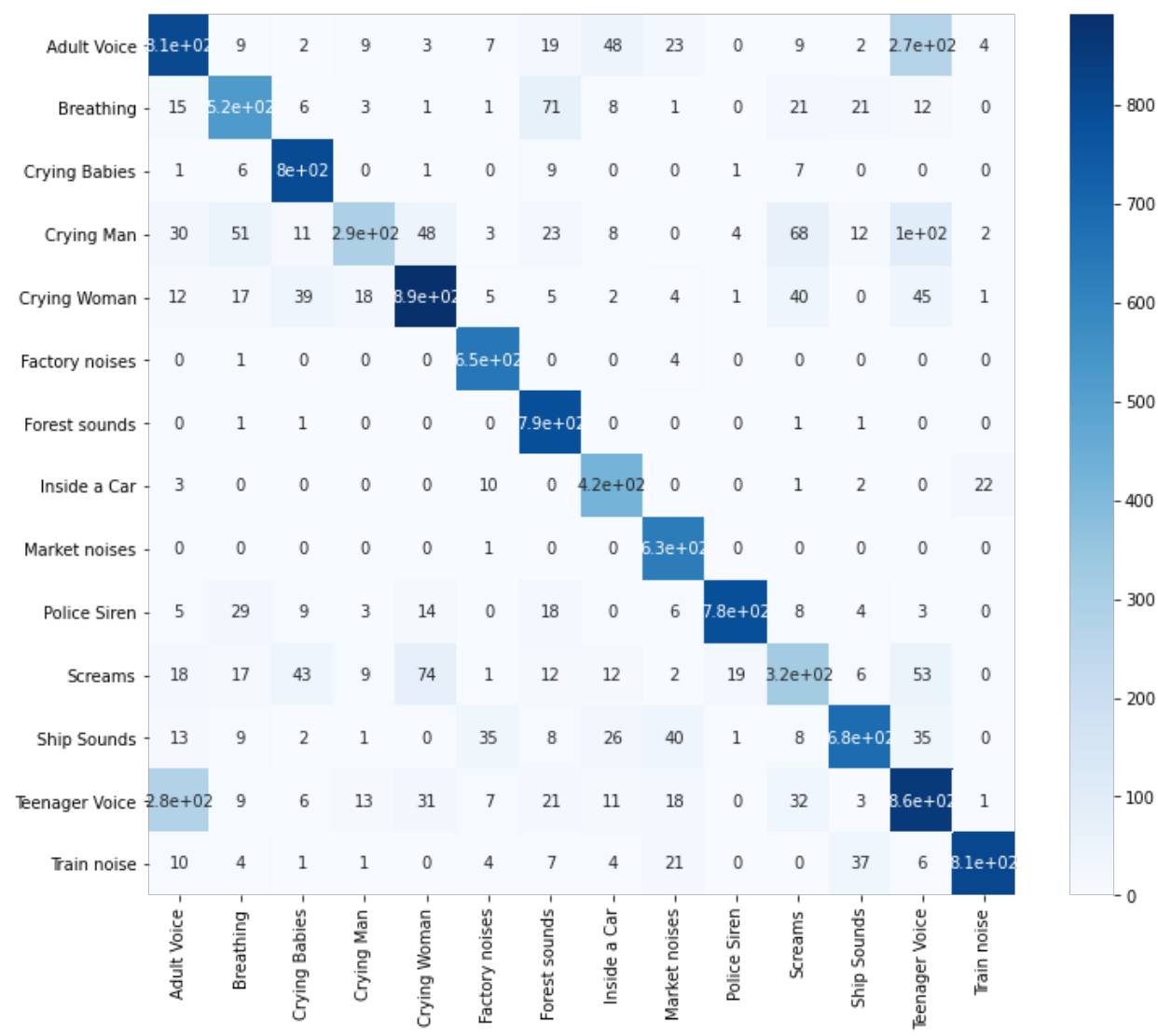

Figure 4.4: Confusion Matrix of the Customized 2D CNN model
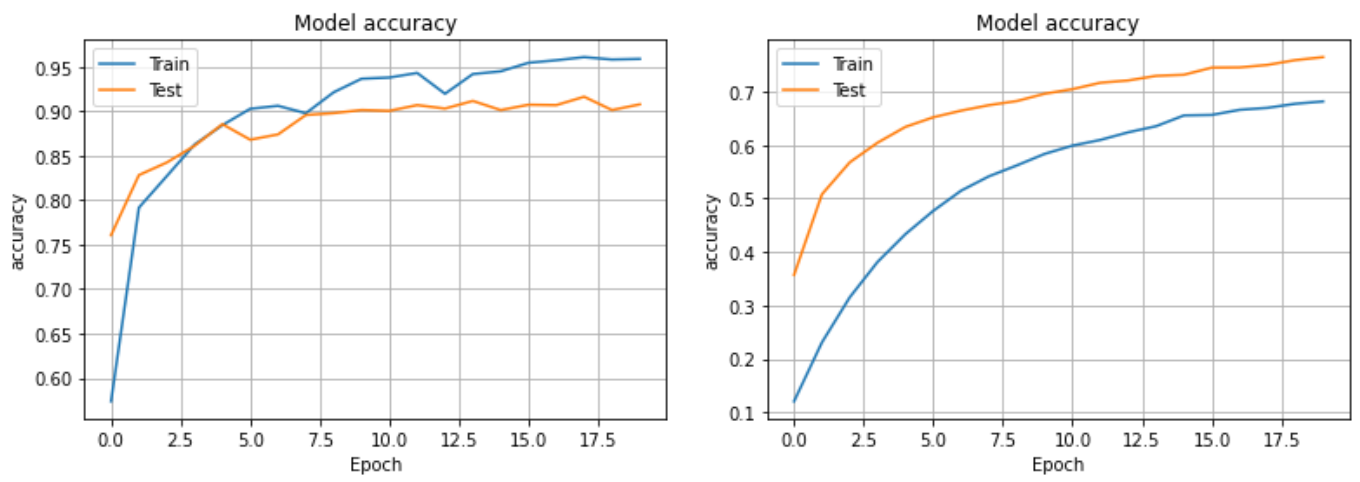

Figure 4.5: The training and validation accuracy and loss of the Customized 2D CNN model 

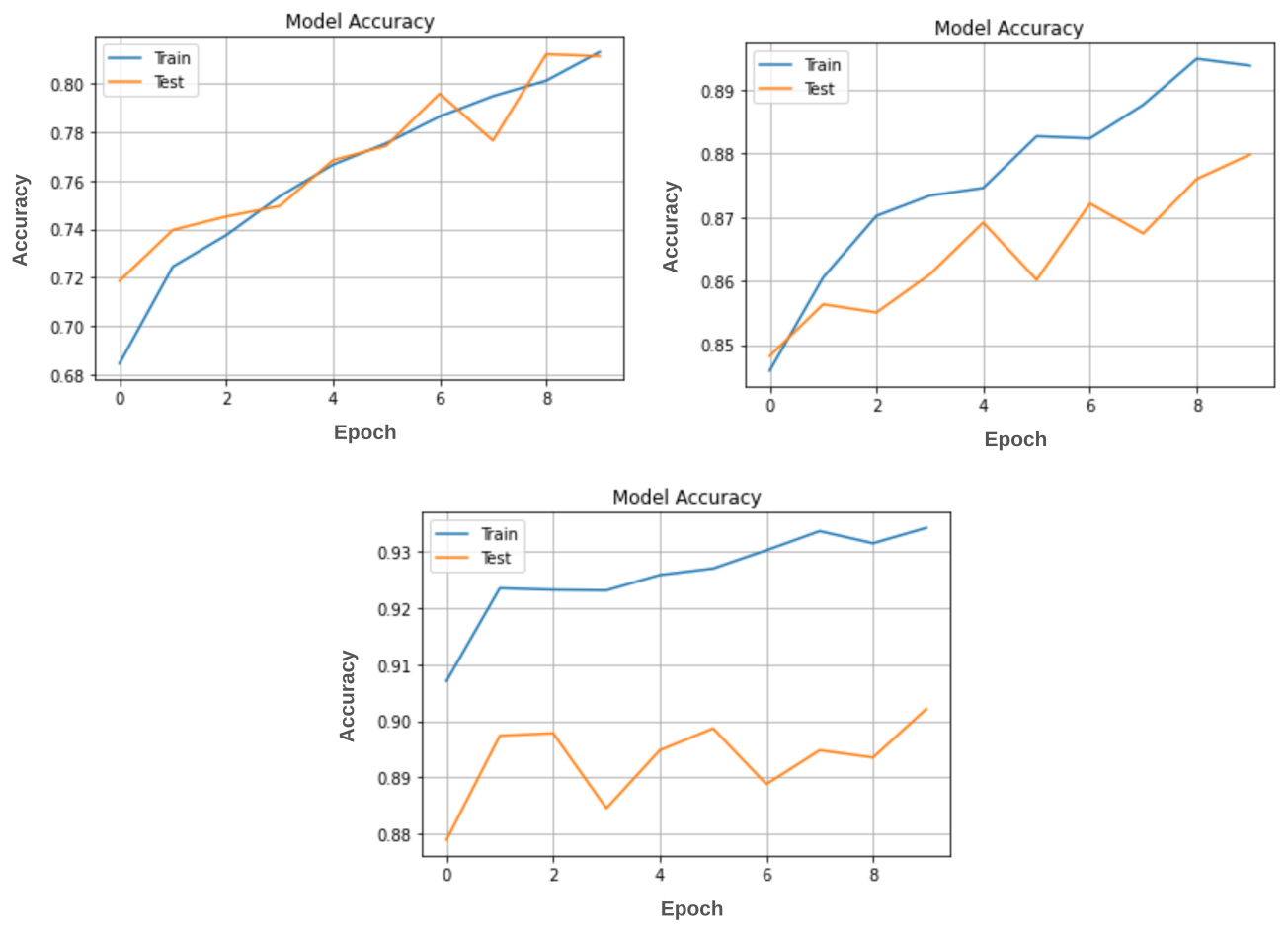

Figure 4.6: Training accuracy and Validation Accuracy per Epochs of LSTM MODEL at each 10 epochs of 30 epochs

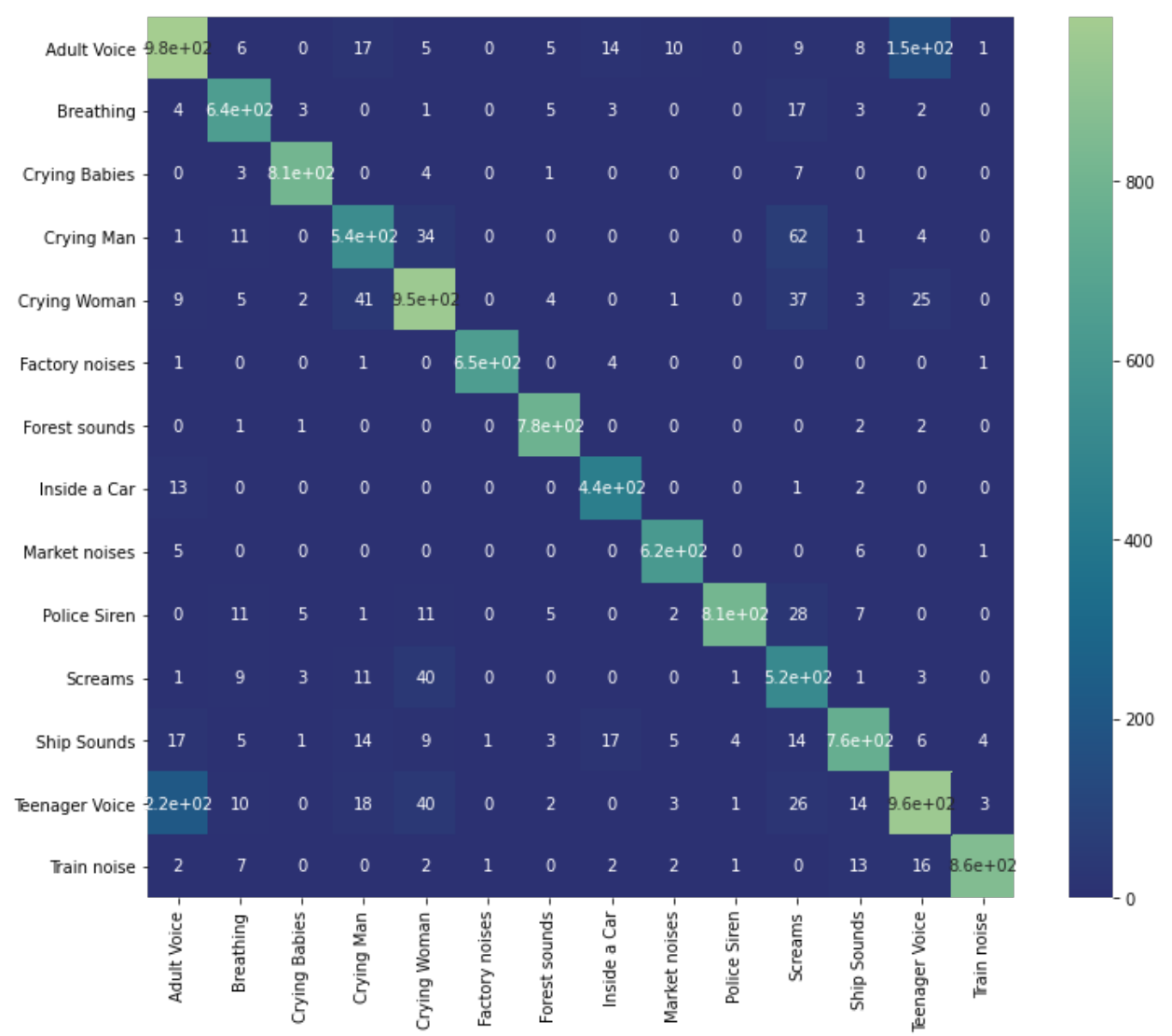

Figure 4.7: Confusion Matrix of LSTM 


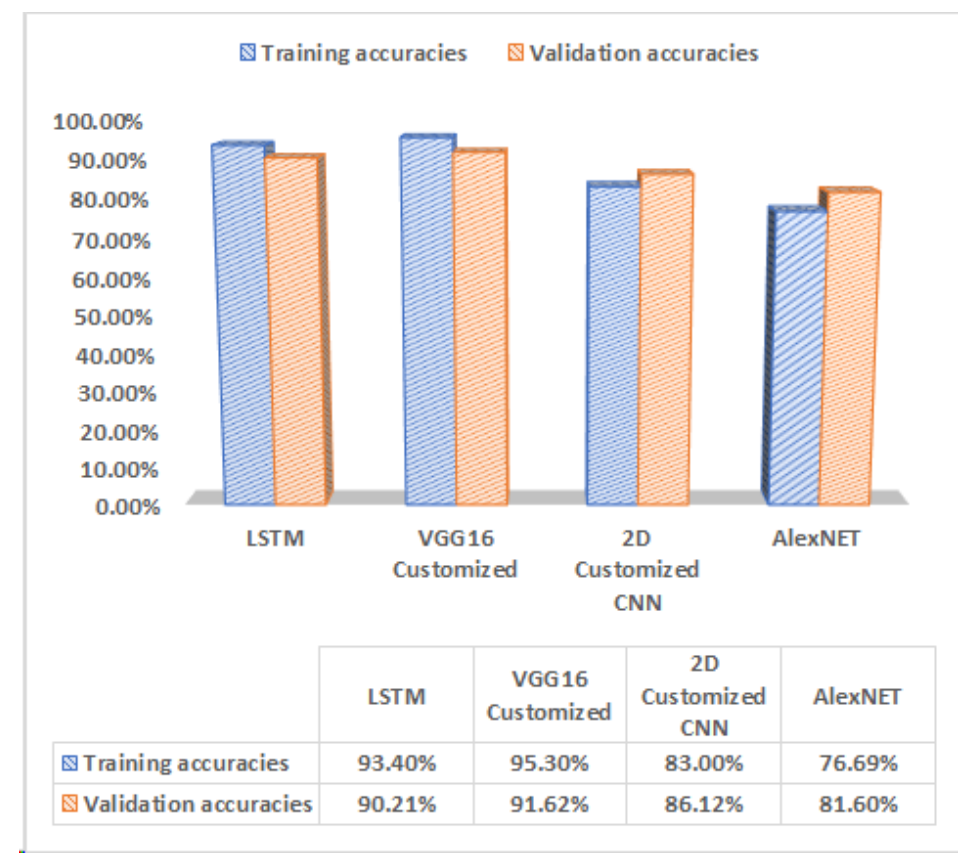

Figure 4.8: Bar chart representing maximum accuracies

Finally, to compare prediction accuracies of each model, we have used a classification report table in Fig 4.8 to summarize the performances of each model in terms of Precision, Recall, and F1 Score respectively. The classification report in table 1 demonstrates the accuracy of the models in terms of predicting the correct classes concerning the testing dataset. It again shows the success of our model in predicting the testing data. 


\begin{tabular}{|c|c|c|c|c|c|c|c|c|c|c|c|c|c|c|c|c|c|c|}
\hline Models & Class & $\begin{array}{l}\text { Adult } \\
\text { Voice }\end{array}$ & Breathing & $\begin{array}{l}\text { Crying } \\
\text { Babies }\end{array}$ & $\begin{array}{l}\text { Crying } \\
\text { Man }\end{array}$ & $\begin{array}{l}\text { Crying } \\
\text { Woman }\end{array}$ & $\begin{array}{l}\text { Factory } \\
\text { Noises }\end{array}$ & $\begin{array}{l}\text { Forest } \\
\text { Sounds }\end{array}$ & $\begin{array}{c}\text { Inside a } \\
\text { Car }\end{array}$ & $\begin{array}{l}\text { Market } \\
\text { Noises }\end{array}$ & $\begin{array}{c}\text { Police } \\
\text { Siren }\end{array}$ & Screams & $\begin{array}{l}\text { Ship } \\
\text { Sounds }\end{array}$ & $\begin{array}{l}\text { Teenager } \\
\text { Voice }\end{array}$ & $\begin{array}{c}\text { Train } \\
\text { Noises }\end{array}$ & Accuracy & Macro Avg & $\begin{array}{l}\text { Weighted } \\
\text { Avg }\end{array}$ \\
\hline \multirow{4}{*}{$\begin{array}{c}\text { VGG16 } \\
\text { (Customized) }\end{array}$} & Precision & 0.86 & 0.98 & 0.97 & 0.84 & 0.95 & 1.00 & 0.98 & 0.93 & 0.95 & 0.99 & 0.77 & 0.92 & 0.76 & 0.99 & - & 0.92 & 0.91 \\
\hline & Recall & 0.71 & 0.94 & 1.00 & 0.87 & 0.86 & 1.00 & 1.00 & 0.98 & 0.99 & 0.98 & 0.90 & 0.90 & 0.86 & 0.96 & . & 0.93 & 0.91 \\
\hline & F1-score & 0.78 & 0.96 & 0.98 & 0.86 & 0.90 & 1.00 & 0.99 & 0.96 & 0.97 & 0.98 & 0.84 & 0.91 & 0.81 & 0.98 & 0.91 & 0.92 & 0.91 \\
\hline & Support & 1213 & 683 & 823 & 654 & 1081 & 655 & 791 & 461 & 633 & 883 & 587 & 861 & 1287 & 907 & 11519 & 11519 & 11519 \\
\hline \multirow{4}{*}{ LSTM } & Precision & 0.82 & 0.94 & 0.98 & 0.89 & 0.91 & 0.97 & 0.99 & 0.98 & 0.96 & 0.99 & 0.74 & 0.93 & 0.77 & 0.97 & - & 0.92 & 0.91 \\
\hline & Recall & 0.78 & 0.94 & 0.99 & 0.80 & 0.88 & 1.00 & 0.99 & 0.90 & 0.99 & 0.95 & 0.88 & 0.89 & 0.84 & 0.97 & - & 0.91 & 0.91 \\
\hline & F1-score & 0.80 & 0.94 & 0.99 & 0.84 & 0.89 & 0.98 & 0.99 & 0.94 & 0.98 & 0.97 & 0.80 & 0.91 & 0.80 & 0.97 & 0.91 & 0.91 & 0.91 \\
\hline & Support & 1213 & 683 & 823 & 654 & 1081 & 655 & 791 & 461 & 633 & 883 & 587 & 861 & 1287 & 907 & 11519 & 11519 & 11519 \\
\hline \multirow{4}{*}{$\begin{array}{l}\text { 2D Customized } \\
\text { CNN }\end{array}$} & Precision & 0.78 & 0.86 & 0.98 & $\overline{0.84}$ & 0.92 & 0.97 & 0.92 & $\overline{0.87}$ & 0.88 & 0.99 & 0.70 & 0.92 & 0.73 & 0.98 & - & 0.88 & 0.88 \\
\hline & Recall & 0.71 & 0.88 & 0.98 & 0.76 & 0.86 & 1.00 & 1.00 & 0.98 & 1.00 & 0.94 & 0.80 & 0.86 & 0.75 & 0.94 & . & 0.89 & 0.88 \\
\hline & F1-score & 0.74 & 0.87 & 0.98 & 0.80 & 0.89 & 0.98 & 0.96 & 0.92 & 0.94 & 0.96 & 0.75 & 0.89 & 0.74 & 0.96 & 0.88 & 0.88 & 0.88 \\
\hline & Support & 1213 & 683 & 823 & 654 & 1081 & 655 & 791 & 461 & 633 & 883 & 587 & 861 & 1287 & 907 & 11519 & 11519 & 11519 \\
\hline \multirow{4}{*}{ AlexNET } & Precision & 0.73 & 0.54 & 0.93 & 0.85 & 0.88 & 0.91 & 0.91 & 0.83 & 0.76 & 0.96 & 0.68 & $\overline{0.92}$ & 0.64 & 0.99 & - & 0.82 & 0.82 \\
\hline & Recall & 0.63 & 0.90 & 0.94 & 0.52 & 0.83 & 0.99 & 0.98 & 0.96 & 1.00 & 0.86 & 0.54 & 0.79 & 0.68 & 0.89 & - & 0.82 & 0.81 \\
\hline & F1-score & 0.68 & 0.68 & 0.93 & 0.65 & 0.85 & 0.95 & 0.94 & 0.89 & 0.86 & 0.91 & 0.60 & 0.85 & 0.66 & 0.94 & 0.81 & 0.81 & 0.81 \\
\hline & Support & 1213 & 683 & 823 & 654 & 1081 & 655 & 791 & 461 & 633 & 883 & 587 & 861 & 1287 & 907 & 11519 & 11519 & 11519 \\
\hline
\end{tabular}

Figure 4.9: Classification Report of VGG16, LSTM, 2D Customized CNN and AlexNet respectively 


\section{Chapter 5}

\section{Conclusion}

This paper proposes models for a thorough investigation of ransom calls. Here, we have used LSTM and the Customized 2D CNN to detect the sounds of ransom calls and later compared their output with established VGG16 and AlexNet output. These models can effectively demystify the different layers of noises present in a ransom phone call, thus unveiling the surroundings of the caller. Additionally, this paper proves the validity of the aforementioned models over other models like vgg16 and AlexNet.

In the future, further analysis will be done as the lstm model and the AlexNet model will be trained and their corresponding output will be visualized. The future scope of this work includes but is not limited to: audio forensic result verification in crime investigation, accident investigation via artificial intelligence, automated audio to text conversion, and noise pollution sound source classification. 


\section{Chapter 6}

\section{Bibliography}

[1] Soothill, K., Francis, B., Ackerley, E. (2007). Kidnapping: A criminal profile of persons convicted 1979-2001. Behavioral sciences the law, 25(1), 69-84.

[2] Concern, A. G. (2019). 15 Ransom Kidnapping. International and Transnational Crime and Justice, 92.

[3] Pires, S. F., Guerette, R. T., Stubbert, C. H. (2014). The crime triangle of kidnapping for ransom incidents in Colombia, South America: A 'litmus' test for situational crime prevention. British Journal of Criminology, 54(5), 784-808.

[4] Noor-Mohamed, Mohd. (2014). The Definitional Ambiguities of Kidnapping and Abduction, and its Categorisation: The Case for a More Inclusive Typology. Howard Journal of Criminal Justice. 53. 10.1111/hojo.12028.

[5] Krizhevsky, A., Sutskever, I., Hinton, G. E. (2012). Imagenet classification with deep convolutional neural networks. In Advances in neural information processing systems (pp. 1097-1105).

[6] Simonyan, K., Zisserman, A. (2014). Very deep convolutional networks for large-scale image recognition. arXiv preprint arXiv:1409.1556.

[7] Chen, H., Zhang, Y., Kalra, M. K., Lin, F., Chen, Y., Liao, P., .. Wang, G. (2017). Low-dose CT with a residual encoder-decoder convolutional neural network. IEEE transactions on medical imaging, 36(12), 2524-2535.

[8] Anthimopoulos, M., Christodoulidis, S., Ebner, L., Christe, A., Mougiakakou, S. (2016). Lung pattern classification for interstitial lung diseases using a deep convolutional neural network. IEEE transactions on medical imaging, 35(5), 1207-1216. 
[9] Ding, C., Tao, D. (2015). Robust face recognition via multimodal deep face representation. IEEE Transactions on Multimedia, 17(11), 2049-2058.

[10] Sun, Y., Chen, Y., Wang, X., Tang, X. (2014). Deep learning face representation by joint identification-verification. In Advances in neural information processing systems (pp. 1988-1996).

[11] Chamoso, P., Raveane, W., Parra, V., González, A. (2014). UAVs applied to the count-ing and monitoring of animals. In Ambient intelligence-software and applications (pp. 71-80). Springer, Cham.

[12] Xu, Y., Yu, G., Wang, Y., Wu, X., Ma, Y. (2017). Car detection from lowaltitude UAV imagery with the faster R-CNN. Journal of Advanced Transportation, 2017.

[13] Hoy, M. B. (2018). Alexa, Siri, Cortana, and more: an introduction to voice assistants. Medical reference services quarterly, 37(1), 81-88.

[14] J. Salamon and J. P. Bello, "Deep Convolutional Neural Networks and Data Augmentation for Environmental Sound Classification," in IEEE Signal Processing Letters, vol. 24, no. 3, pp. 279-283, March 2017, doi: 10.1109/LSP.2017.2657381.

[15] M. Tschannen, T. Kramer, G. Marti, M. Heinzmann and T. Wiatowski, "Heart sound clas-sification using deep structured features," 2016 Computing in Cardiology Conference (CinC), Vancouver, BC, 2016, pp. 565-568.

[16] Qian, K., Zhang, Z., Baird, A., Schuller, B. (2017). Active learning for bird sound classification via a kernel-based extreme learning machine. The Journal of the Acoustical Society of America, 142(4), 1796-1804.

[17] Gao, Z., Ben, K., Cui, L. (2009, July). Underwater Vehicle Noise Source Recognition Using Structure Dynamic Adjustable SVM. In 2009 Symposia and Workshops on Ubiquitous, Autonomic and Trusted Computing (pp. 423-427). IEEE.

[18] Bu, S. J., Cho, S. B. (2019, December). Classifying In-vehicle Noise from Multi-channel Sound Spectrum by Deep Beamforming Networks. In 2019 IEEE International Conference on Big Data (Big Data) (pp. 3545-3552). IEEE. 
[19] Thwe, K. Z., War, N. (2017, June). Environmental sound classification based on time-frequency representation. In 2017 18th IEEE/ACIS International Conference on Software Engineering, Artificial Intelligence, Networking and Parallel/Distributed Computing (SNPD) (pp. 251-255). IEEE.

[20] Salamon, J., Bello, J. P. (2017). Deep convolutional neural networks and data augmentation for environmental sound classification. IEEE Signal Processing Letters, 24(3), 279-283.

[21] Amiriparian, S., Gerczuk, M., Ottl, S., Cummins, N., Freitag, M., Pugachevskiy, S., ... Schuller, B. W. (2017, August). Snore Sound Classification Using ImageBased Deep Spectrum Features. In INTERSPEECH (Vol. 434, pp. 3512-3516).

[22] Bahari, M. H., Van Hamme, H. (2011, September). Speaker age estimation and gender detection based on supervised non-negative matrix factorization. In 2011 IEEE Workshop on Biometric Measurements and Systems for Security and Medical Applications (BIOMS) (pp. 1-6). IEEE.

[23] Ali, Z., Imran, M., Alsulaiman, M. (2017). An automatic digital audio authentication/forensics system. IEEE Access, 5, 2994-3007.

[24] Kishore, K. V. K., Satish, P. K.: Emotion recognition in speech using MFCC and wavelet features. In: 3rd IEEE International Advance Computing Conference (IACC), Ghaziabad, 842-847 (2013)

[25] Ioffe, S., Szegedy, C.: Batch normalization: Accelerating deep network training by reducing internal covariate shift, (2015)

[26] Srivastava, N., Hinton, G., Krizhevsky, A., Sutskever, I., Salakhutdinov, R. (2014). Dropout: a simple way to prevent neural networks from overfitting. The journal of machine learning research, 15(1), 1929-1958.

[27] Bashar, A. (2019). SURVEY ON EVOLVING DEEP LEARNING NEURAL NETWORK ARCHITECTURES. Journal of Artificial Intelligence, 1(02), 73-82.

[28] Varshni, D., Thakral, K., Agarwal, L., Nijhawan, R., Mittal, A. (2019, October 
17). Pneumonia Detection Using CNN based Feature Extraction.

[29] Bahari and H. Van Hamme, "Speaker age estimation and gender detection based on supervised Non-Negative Matrix Factorization," 2011 IEEE Workshop on Biometric Measurements and Systems for Security and Medical Applications (BIOMS), Milan, 2011, pp. 1-6, doi: 10.1109/BIOMS.2011.6052385.

[30] Amiriparian, S., Gerczuk, M., Ottl, S., Cummins, N., Freitag, M., Pugachevskiy, S., ... Schuller, B. W. (2017, August). Snore Sound Classification Using ImageBased Deep Spectrum Features. In INTERSPEECH (Vol. 434, pp. 3512-3516)

[31] Ali.Z, M. Imran and M. Alsulaiman, "An Automatic Digital Audio Authentication/Forensics System," in IEEE Access, vol. 5, pp. 2994-3007, 2017, doi: 10.1109/ACCESS.2017.2672681. 